\title{
THE EFFECTS OF DOMINANCE ON SOCIAL FORAGING TACTIC USE IN HOUSE SPARROWS
}

\author{
by
}

\begin{abstract}
ANDRÁS LIKER ${ }^{1,2)}$ and ZOLTÁN BARTA ${ }^{3,4,5)}$
$\left({ }^{1}\right.$ Behavioural Biology Research Group, Department of Ecology, Faculty of Veterinary Sciences, Szent István University, Pf. 2, H-1400, Budapest, Hungary; ${ }^{3}$ Behavioural Ecology Research Group, Department of Evolutionary Zoology and Human Biology, University of Debrecen, Pf. 3, H-4010 Debrecen, Hungary)
\end{abstract}

(Acc. 14-VI-2002)

\section{Summary}

We investigated whether social foraging tactic use (producing and scrounging) in birds is affected by the dominance rank of individuals as predicted by a phenotype limited producerscrounger game. In a captive flock of house sparrows, we observed the behaviour of the birds when they were foraging on a grid containing clumps of seeds. We measured the fighting success of the birds, determined the method by which they found food clumps (finding or joining), and measured their feeding rate. Joining were frequently observed and usually involved aggressive interactions. Most birds used both finding and joining to obtain food. We found that foraging method was related to dominance: the frequency of joining gradually increased with increasing dominance rank, as predicted by the phenotype limited model for flocks where there are moderate competitive asymmetries among the birds. Food intake rate of individuals was not related to either their dominance rank or foraging method. Similar weak relationships were predicted by the model among these variables for flocks with moderate competitive asymmetries. Behavioural variability among sparrows in locomotion frequency and vigilance was not related to their foraging method, but the rate of investigating potential

2) Corresponding author's e-mail address: aliker@univet.hu

4) Present address: Centre for Behavioural Biology, School of Mathematics, University of Bristol, University Walk, Bristol BS8 1TW, UK.

5) We thank I. Coolen, C.A. Johnson and an anonymous referee for their valuable comments on the manuscript. We also thank students at the Department of Ecology, Szent István University, for their assistance in capturing and transporting birds. A.L. and B.Z. were supported both by Bolyai János Research Fellowships and Békésy György Postdoctoral Fellowships. The study was supported by OTKA grants F026595 to A.L. and T030434 to B.Z. 
food caches strongly decreased with increasing frequency of joining. We conclude that the phenotype limited model successfully predicts the relationship between dominance and joining frequency in house sparrows feeding on concentrated food sources, and we suggest that the distribution of food used in tests of the model may crucially affect their results.

\section{Introduction}

Birds frequently forage in flocks, and a common form of intra-flock interactions is when some of the group members exploit the food finding efforts of others. In ground feeding passerines, for instance, some individuals actively search for food patches (producers) while others (scroungers) wait for producers to discover a patch and then feed from it (Barnard \& Sibly, 1981; Giraldeau \& Caraco, 2000). In such producer-scrounger systems, the foraging payoffs to flock members are affected both by the forager's own behaviour and that of its companions (i.e. frequency dependence), and can only be analyzed by game theoretical approaches (Giraldeau \& Caraco, 2000). The models developed to investigate producer-scrounger systems focus on the stable frequencies of the alternative foraging tactics, the payoffs of social foragers, and the ecological determinants of optimal tactic choice. Recent empirical tests show that producer-scrounger models can successfully predict foraging tactic use in some ground feeding birds (Giraldeau \& Caraco, 2000; Mottley \& Giraldeau, 2000; Coolen et al., 2001).

In several species, individuals often behave aggressively during feeding, and may form dominance hierarchies within the foraging flocks. Because the dominance rank of individual s may crucially affect their success in competition for food, dominance relationships are expected to influence social foraging tactic use (Giraldeau \& Beauchamp, 1999). To understand how an individual's dominance rank influences its use of producer or scrounger tactics, Barta \& Giraldeau (1998) developed a phenotype limited producerscrounger model in which they assumed asymmetries in competitive abilities (closely correlated with dominance rank) among birds feeding in small flocks. The first prediction of this model is that dominant individuals play scrounging tactic more often than subordinates, with differences in tactic use depending on the degree of competitive asymmetries among the flock members. For example, when competitive asymmetries are high (i.e. when dominants compete for food much more successfully than subordinates), tactic use changes as a step function of dominance rank: high ranking dominants 
exclusively use scrounger and the others play producer. For a moderate degree of competitive asymmetries the model predicts less differences in tactic use: all birds may use both tactics, and scrounging frequency increases gradually with increasing dominance rank. The second prediction of the model is that the payoffs of individuals are unequal: individuals being dominant and playing scrounger tend to have higher food intake rates than subordinate producers, especially when competitive asymmetries are strong. There is some empirical evidence, e.g. in dark-eyed juncos (Junco hyemalis), whitethroated sparrows (Zonotrichia albicollis) and Harris' sparrows (Zonotrichia querula) that dominant individuals preferentially use the scrounger strategy (Baker et al., 1981; Rohwer \& Ewald, 1981; Theimer, 1987; Caraco et al., 1989; Wiley, 1991). Note, however, that most of these studies either restricted their examination to too few individual s (i.e. dyads or trios; Theimer, 1987; Caraco et al., 1989), or did not explicitly test an individual's tactic use (e.g. Rohwer \& Ewald, 1981; Wiley, 1991). On the other hand, the results of some other studies do not support the model's predictions (Barnard \& Sibly, 1981; Giraldeau et al., 1990; Biondolillo et al., 1997). Thus, although the theory predicts that dominance may have important effects on tactic use in producer-scrounger systems, the empirical tests of the problem provided conflicting results so far. In general, the phenotype limitation of foraging tactic use is poorly known in birds (Giraldeau \& Beauchamp, 1999).

In this study we investigated the effects of dominance on foraging tactic use in a captive flock of house sparrows. House sparrows are an ideal candidate because winter flocks exhibit a well defined dominance hierarchy, the birds compete aggressively for food and they have been shown to use producer-scrounger tactics (Barnard \& Sibly, 1981; Møller, 1987; Johnson et al., 2001; Liker \& Barta, 2001). In a previous study, Barnard \& Sibly (1981) reported that, contrary to expectation, subordinate house sparrows might have played scrounging. Note, however, that Barnard and Sibly provided food for the birds in large and scarce patches, and that mostly nonaggressive forms of scrounging (area copying and following) were used by the birds. Competitive asymmetries may affect foraging when the superior competitors (dominants) decide to defend food patches aggressively, leading to the exclusion of subordinate individuals. Thus, the effects of dominance on tactic use are expected to be stronger and more detectable when individuals forage on aggregated and abundant food patches (Barta \& Giraldeau, 1998). In this study, therefore, we offered highly clumped and rich food sources 
to foraging sparrows to promote aggressive competition for food and then tested how dominance is related to their feeding method under such conditions.

Specifically, we had two aims. First, we used observation s to investigate the effects of dominance on foraging tactic use, and compared these correlative results to the patterns predicted by the phenotype limited model (Barta \& Giraldeau, 1998). Although more explicit tests of the model would be conducted by controlled experiments, e.g. by manipulating competitive asymmetries, our main aim here was to describe the basic patterns of aggressive scrounging and to relate the natural variability in dominance to the sparrows' social foraging tactic use.

Second, we studied some elements of the foraging behaviour that may be related to tactic use. Specifically, we investigated whether individual differences in locomotion, vigilance, and exploration of potential food caches are related to the sparrows' tactic use. Discovering behavioural cues that indicate whether individuals are searching for finding or joining opportunitie s could help to quantify the use of the two tactics at a finer scale (Giraldeau \& Beauchamp, 1999; Coolen et al., 2001). For example, some producer-scrounger models predict that individuals using the scrounger tactic may spent more time on scanning their environment than producers (e.g. Ranta et al., 1996, 1998). Indeed, some form of scanning behaviour, e.g. frequencies of hopping with the head pointing up may be associated with the frequencies of a bird's scrounging (Coolen et al., 2001). To our knowledge, other elements of the birds' foraging behaviour have not been analysed in relation to their tactic use.

\section{Methods}

The study flock

We observed the foraging behaviour of sparrows in a captive flock of ten males and five females. The size of the study flock was within the range of natural variation of the house sparrows' flock size (e.g. Barnard, 1980; Elgar \& Catterall, 1981), and also corresponded to the flock size assumed in the model (max. 20 birds; Barta \& Giraldeau, 1998). All birds were captured with mist nets at the 'Dóra-farm' Experimental Station of the Szent István University, $30 \mathrm{~km}$ south-east from Budapest, on 28 September and 4 October 1999. The birds were measured and banded with a metal ring and a unique combination of three colour rings. We also painted small red or white markings on the top of the head or on tail feathers to facilitate quick individual recognition for the observer.

Birds were transferred to the campus of Szent István University, where they were kept in an indoor aviary measuring $3 \times 4 \times 2 \mathrm{~m}$ (height). The aviary was equipped with a $2 \mathrm{~m}$ 
tall roosting tree, a water dish and a sand dish. Feeding took place on a $1.2 \times 1.2 \mathrm{~m}$ wooden feeding-table (hereafter 'grid'), that was placed on the floor of the aviary. For presenting food, the grid contained $144(12 \times 12)$ small holes drilled into the table at $10 \mathrm{~cm}$ intervals. The diameter of these holes was $2.5 \mathrm{~cm}$ and their depth was $1.2 \mathrm{~cm}$. Water and food (a mixture of millet, oat, and sunflower seeds, enriched with vitamins) were provided ad libitum between the observations. We also provided cuttle-bones to supply the birds' lime needs and items of fine gravel to facilitate digestion. Artificial light was provided with 10:14 hr light:dark periods. The birds were released after the experiment at the site of capture on 20 December 1999.

\section{Behavioural data collection}

Birds were observed for a total of 20 observation days between 18 October and 9 December 1999. Observation days were usually separated by periods of non-testing days when birds were fed ad libitum. The evening prior to an observation day, we removed food from the grid and from the floor of the aviary. Feeding trials were conducted the following day. Millet seeds were placed into two clumps consisting of 6 holes on the grid $(N=12$ holes each containing cc. 30 seeds per hole). The locations of the clumps were chosen randomly. Several feeding trials were run in quick succession (typically 5-8 trials per day). The trials were separated by a few minutes, during which new seed clumps were placed on the grid, using new groups of holes for each trial (birds usually depleted the food clumps by the end of a trial). Individuals were observed in a random order within each day.

The birds normally arrived to feed within $1 \mathrm{~min}$ of having placed the seeds on the grid. A randomly chosen focal bird was filmed through the course of a trial, such that only one bird was filmed during a trial. A trial started when the focal individual was recognised from those foraging on the grid and ended when the focal bird left the grid (mean $\pm \mathrm{SE}$ duration: $152.6 \pm 3.8 \mathrm{sec}, N=142$ trials). For each bird, $8-11$ feeding trials were recorded (mean \pm SE: $9.5 \pm 0.2$ trials), and each individual was filmed only once during a particular day.

To describe foraging methods of sparrows, we divided feeding events into two types, finding and joining. These terms were used instead of producing and scrounging, respectively, because we recorded actual feeding events and not directly observed tactic use, i.e. whether a bird was searching as a producer or a scrounger (Mottley \& Giraldeau, 2000; Coolen et al., 2001). We defined a feeding event as joining when the hole from which the focal bird fed was occupied by another feeding bird immediately before, or at the moment of the arrival of the focal individual. We did not consider other types of joining such as area copying (searching for food in holes close to occupied holes), although we recorded when a seed was gained by non-aggressive joining, i.e. was obtained from an occupied hole without aggressive interactions. Finding was defined as a bird discovering an unoccupied hole (i.e. no other bird within $10 \mathrm{~cm}$ from the hole) with seeds in it.

From the video tapes, the following variables were measured for each feeding trial. We defined joining and finding frequency as the number of holes found by joining and finding, respectively, by the focal bird during the trial. Foraging tactic use was then measured by two variables: (1) Proportion of joining: frequency of joining divided by the total number of holes used for feeding by the focal bird during the trial. (We also measured the rate of joining, that was the number of joining per unite time; however, because this latter variable correlated strongly with the proportion of joining, and the two variables gave almost identical results in the analyses, we did not repeat results on joining rate.) (2) Joining attempt rate: 
for this variable we counted the number of attacks performed by the focal bird against foraging individuals (i.e. attempts to take over their feeding holes), and divided it by the time spent on the grid. We calculated this latter variable to measure the behavioural tendencies of individuals independently from their success in joining.

The competitive ability of individuals was also described by two variables. (1) Fighting success: proportion of fights won by the focal bird, expressed as the number of wins divided by the total number of fights in which the focal bird were involved. The focal individual was considered to win a contest if he or she clearly supplanted the opponent (Liker \& Barta, 2001). (2) Defence success: proportion of fights won by the focal bird, including only those fights that was initiated by an opponent. We calculated this latter variable because in most cases joining birds aggressively supplanted others from feeding holes (see below), thus data on overall fighting success were partially non-independent from data on foraging tactic use. By considering success in defence, we reduced this interdependence between fighting and foraging data. Fighting success correlates strongly with dominance ranks in captive house sparrows (Liker \& Barta, 2001).

We measured feeding rate as the number of seeds eaten by the focal bird per unite time, i.e. the number of pecks divided by the time spent on the grid. This measure of feeding rate included all pecks of the focal bird, combining pecks from both finding and joining events. To compare the success of the two different feeding methods, we also calculated the average number of seeds obtained from either joining or finding events (e.g. total number of pecks from joining divided by the number of joining events). To study whether variability in tactic use was associated with changes in some elements of the sparrows' feeding behaviour, we collected data for three further behavioural variables: (1) Frequency of locomotion: for three 10-sec subsamples of the tape of each trial, we counted the number of jumps the focal bird performed to move between potential feeding holes. The start of each $10 \mathrm{sec}$ sample was random with respect to the actual action of the bird, and one sample was taken from each of the first, second and last third of the trial, respectively. The mean value of these three subsamples was then calculated to measure locomotion frequency during the trial. (2) Vigilance: during the same three 10-sec subsamples, we recorded at every exact second (i.e. 10 times per subsample) the position of the bill of the focal bird. Birds were considered being vigilant if their bill was held at or above the horizontal (Coolen et al., 2001). We consider a bird to focus on the feeding ground if the bill was oriented toward the grid, i.e. was held below the horizontal (Coolen et al., 2001). Head position was scored during all activities, so this estimate of vigilance included time when individuals were moving, staying, consuming seeds etc. Vigilance was then measured as the number of records when the birds held their bill above or at horizontal. The mean value of the three subsamples was calculated to measure vigilance during the whole trial. Birds were videotaped from cc. $2 \mathrm{~m}$ distance, so in most cases they were taped from side and the head position was visible despite the fact that the feeder was placed on the floor. (3) Food searching rate: we counted the number of holes investigated for food by the focal bird. We defined a bird as to investigate a hole when he or she clearly looks into the hole, e.g. by quick glances into the hole with characteristic side-turned head position or by digging the head into the hole. Food searching rate was then calculated as the number of investigated holes divided by the time spent on the grid.

Finally, three times during each trial we also recorded the number of birds present on the grid, and we used the mean of the three counts to measure bird density. There was no significant difference, however, between individuals in the number of birds present on the table during their trials (mean \pm SE: $11.0 \pm 0.2$ birds; Kruskal-Wallis test, $\chi^{2}=18.2$, 
$\mathrm{df}=14, p=0.20$ ), so we did not included bird density in the data analyses. Time of the day did also not influence the behaviour of birds during the trials (Kruskal-Wallis tests, pecking rate: $\chi^{2}=5.1, \mathrm{df}=8, p>0.7$; proportion of joining: $\chi^{2}=2.7, \mathrm{df}=8, p>0.9$; number of fights per trial: $\chi^{2}=6.2 \mathrm{df}=8, p>0.6$; and $p>0.1$ for all other behaviour variables).

\section{Statistical analyses}

We used Spearman rank correlations $\left(r_{s}\right)$ to analyse relationships between individuals' dominance, their tactic use, feeding success, and the recorded behavioural variables. For this purpose we used individuals as independent data points, i.e. the mean values of the trials were used for each bird. When we compared mean seed numbers obtained from finding and joining events we also took mean values for each individual. In the cases of multiple comparisons, we applied Bonferroni corrections to keep Type I errors at $\alpha=0.05$ level (Motulsky, 1995). Mean \pm SE and uncorrected two-tailed probabilities are given, and we noted when the Bonferroni correction of significance level altered the conclusion of the statistical tests.

\section{Results}

\section{Individual differences in the foraging method}

Both finding and joining were frequently used by house sparrows to gain access to food patches (mean \pm SE number of holes per trial obtained by finding: $7.0 \pm 0.4$, and by joining: $1.3 \pm 0.2, N=142$ trials). Most joining events involved aggressive interaction s between two individuals: the bird using joining attacked a feeding individual and supplanted him or her from the hole containing the food. A particular hole could be defended by the feeding individual, but the whole patches of six food-containing holes were never defended by a feeding individual. Non-aggressive joining such as shared use of a hole by two birds were rarely observed. Similarly, in a few cases, birds attempted to get seeds from an occupied hole by a single, quick pecking into the hole, without aggression, and they usually left the hole immediately. In general, these two forms of non-aggressive joining occurred with low frequencies $(4.7 \%$ of seeds pecked from scrounged holes was gained in non-aggressive ways).

Most individuals used both finding and joining to obtain food, although there were large individual differences in the relative frequencies of these two types of feeding events (Fig. 1a). The proportion of food patches gained by joining ranged from $0 \%$ to $47 \%$ (one male did not use joining during the observations), and the average proportion of joining was $16.7 \pm 3.9 \%$ for the entire flock $(N=15)$. Individuals using joining with high frequencies 
(a)

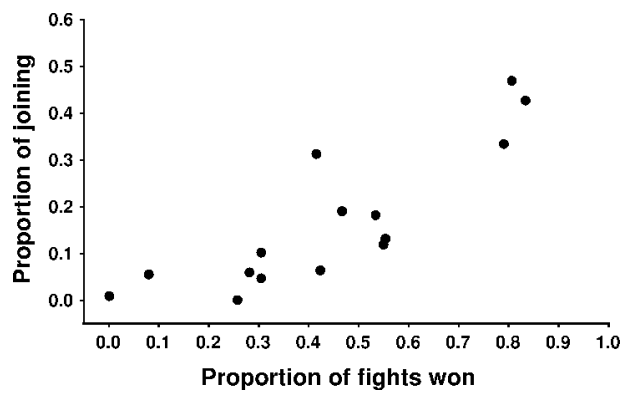

(b)

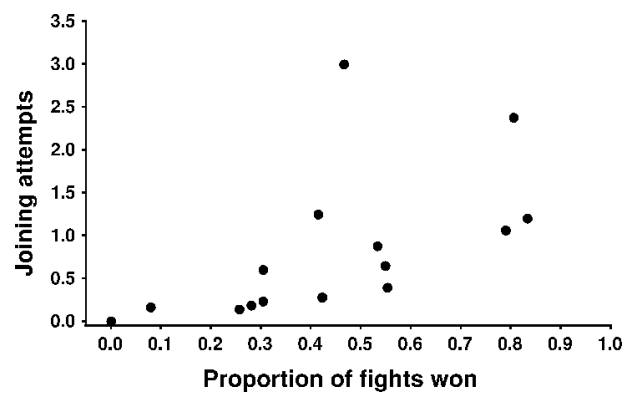

Fig. 1. The relationships between fighting success of individuals and (a) the proportion of joining, and (b) the rate of aggressive joining attempts (measured as the number of attacks on feeding birds per min).

seemed to have periods when they searched for unoccupied food patches, and for other periods they changed to joining and continuously attacked other individuals. There was no significant differences between the sexes in the proportion of joining (males: $20.6 \pm 5.4 \%$, females: $9.1 \pm 2.5 \%$, MannWhitney $U$-tests: $U=15.0, p=0.22, N=15)$.

\section{Joining versus dominance}

A high proportion of individual variation in the occurrence of joining events was explained by differences in the competitive ability of birds. First, fighting success (proportion of fights won) was strongly correlated with the proportion of joining $\left(r_{s}=0.86, p<0.001\right.$; Fig. 1a). Since data on fighting success and proportion of joining were collected non-independently (they were measured from the same trials, and joining frequently involved successful attacks) we repeat this analysis to eliminate interdependence as follows. First, we divided the trials of each individual randomly into two groups (e.g. 10 trials of the same bird were divided into two groups with 
5 trials in each). Then we calculated fighting success of the individual from one group of the trials (e.g. from the first 5 randomly chosen trials) and proportion of joining from the other group of trials (e.g. from the other 5 trials). This procedure ensured that the two variables were estimated independently for each bird. Then a Spearman correlation was calculated between fighting success and the proportion of joining for this data set. The entire procedure was repeated 1000 times. The median $r_{s}$ of these replications was 0.69 and their median $p$ value was 0.005 . These findings indicate that the correlation found between the fighting success and the proportion of joining is not a by-product of their interdependent estimation. Fighting success also correlated strongly with the rate of joining attempts $\left(r_{s}=0.77, p=0.001\right.$, Fig. $1 \mathrm{~b}$; for the randomly divided data sets median $r_{s}=0.66$, median $p=0.009$ ). So dominant birds were not only more successful in using joining but they preferred to use joining as compared to subordinates. The sexes did not differ significantly in their fighting success (males: $49.6 \pm 8.6 \%$, females: $33.0 \pm 7.5 \%$, Mann-Whitney $U$-test: $U=$ $16.0, p=0.27, N=15$ ).

Second, when we used defence success to describe competitive ability of individuals (a measure independent of success in joining, see Methods), this measure of dominance also correlated strongly with the proportion of joining $\left(r_{s}=0.65, p=0.009\right)$, but was not significantly related to the rate of joining attempts $\left(r_{s}=0.51, p=0.054\right.$; Bonferroni correction for four comparisons, critical $\alpha=0.0127$ ).

As different measures of dominance and tactic use were interrelated and tended to give the same result, only the fighting success and the proportion of joining were used in the further analyses.

\section{Feeding success}

Individuals that obtained a high proportion of holes by joining tended to have higher overall feeding rates (number of pecking per unite time) than those obtained food patches mostly or exclusively by finding, although the correlation was not significant after correction $\left(r_{s}=0.53, p=0.044\right.$; critical $\alpha=0.0253$ for two comparisons). Feeding rate was not related significantly to dominance measured as fighting success $\left(r_{s}=0.39, p=\right.$ $0.16)$. We found no significant differences in the mean number of seeds eaten per hole between finding events $(6.2 \pm 0.7$ pecking per hole $)$ and joining 


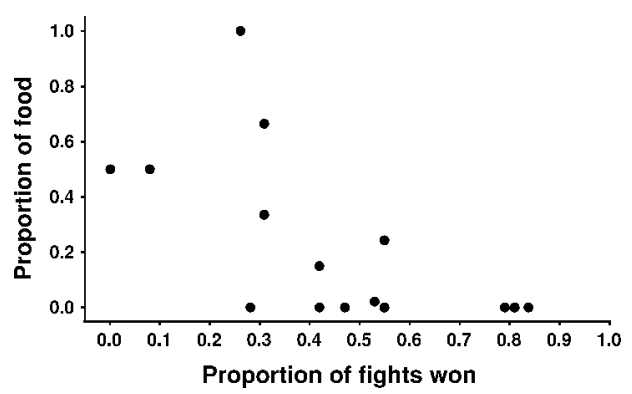

Fig. 2. The relationship between fighting success of individuals and the proportion of scrounged food items that was obtained by non-aggressive joining $(1=$ all joined food items obtained by non-aggressive joining, $0=$ all joined food items obtained by aggressive joining).

events $(8.1 \pm 1.1$ pecking per hole; Wilcoxon matched-pairs signed-ranks test, $z=1.35, p=0.18, N=14$; one male that used finding exclusively was omitted). From the seeds obtained by joining, the proportion gained by non-aggressive forms of joining strongly decreased as the fighting success of individuals increased ( $r_{s}=-0.69, p=0.005$; Fig. 2$)$, i.e. subordinates mainly gained their scrounged food items by avoiding aggressive interaction $s$ with others.

\section{Behavioural correlates of joining}

The relationships between feeding method, dominance and the measured behavioural variables are shown in Table 1. Locomotion frequency measured as the number of jumps during $10 \mathrm{sec}$ samples was not related either to the fighting success of birds or to the proportion of joining. Similarly, vigilance behaviour was unrelated to fighting success and to the proportion of joining. Food searching rate was also not related to dominance, but there was a strong negative correlation between food searching rate and the proportion of joining (Table 1, Fig. 3). Thus, individuals with low proportion of joining were more likely to investigate holes for food clumps than those frequently obtaining food by joining.

\section{Discussion}

We found a strong association between dominance and foraging method in house sparrows: dominants obtained food by joining more often than sub- 
TABLE 1. Variability in three elements of the house sparrows' foraging behaviour in relation to the dominance and foraging tactic use of the individuals

\begin{tabular}{lccc}
\hline & $\begin{array}{c}\text { Locomotion } \\
\text { frequency }\end{array}$ & Vigilance & $\begin{array}{c}\text { Food searching } \\
\text { rate }\end{array}$ \\
\hline Fighting success & & & -0.42 \\
$r_{s}$ & -0.25 & 0.13 & 0.12 \\
$p$ & 0.37 & 0.65 & -0.68 \\
Proportion of joining & -0.30 & 0.24 & $0.006^{*}$ \\
$r_{s}$ & 0.28 & 0.40 & \\
$p$ & & & \\
\hline
\end{tabular}

Dominance was measured as fighting success, whereas tactic use was measured as the proportion of joining. The table shows Spearman rank correlation coefficients and the corresponding uncorrected $p$ values ( $N=15$ individuals in all correlations). Critical $\alpha$ was set at $\alpha=0.0085$, according to Bonferroni correction for six comparisons, and asterisk marks significant correlation after the correction.

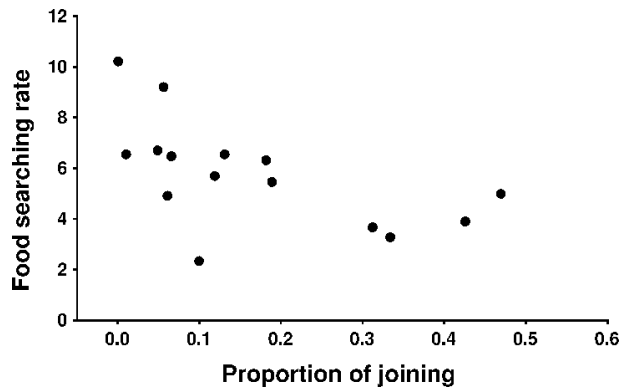

Fig. 3. The relationship between foraging tactic use (proportion of joining) and the rate of food searching (number of holes investigated per min).

ordinates. We showed that this relationship was robust, i.e. it remained unchanged when different measures were used to estimate both dominance and foraging method, and when fighting success and joining were estimated from different trials. It is clear that dominants should have more success in joining when this tactic involves aggressive supplantation of other individuals from food patches, as we observed in most cases in house sparrows. In addition, our study showed that subordinates were not only less successful but also less likely to attempt aggressive joining than dominants. This reduction in the frequency of joining by subordinates might have helped them to save efforts from unsuccessful feeding attempts, because they were easily driven 
from food patches occupied by other birds. Our results contrast those reported by Barnard \& Sibly (1981), who suggested an opposite relationship s between dominance and some forms of joining. We suggest that the main factor responsible for the differences between the two studies is the distribution and value of food for which the sparrows competed during the experiments. In Barnard \& Sibly's (1981) study sparrows searched on a grid for holes which contained a single piece of mealworm, while we presented seeds clumps in the holes. Because seed clumps represented rich food sources, our study set-up stimulated intense competition for the food patches, including the use of the aggressive joining. On the other hand, our results showed that subordinates obtained their scrounged food items mostly in non-aggressive ways. If non-aggressive joining is also played mostly by subordinates when food concentrations is low (i.e. when aggressive joining is unprofitable), than this difference in the use of different forms of joining between individuals of different competitive abilities may explain the higher joining rate of subordinates reported by Barnard \& Sibly (1981).

Similar to some other passerine species, most house sparrows used both feeding tactics to find food (e.g. Giraldeau et al., 1994; Giraldeau \& Livoreil, 1998), and some dominant individuals seemed to alternate between periods of successive finding attempts and periods of successive joining attempts. There was no step change in tactic use at some critical dominance rank in the flock, as predicted by the Barta \& Giraldeau's (1998) model for high between-individual competitive asymmetries. Instead, the proportion of joining increased gradually with increasing competitive ability of individuals. This pattern conforms most closely to the relationship predicted by the model for 'intermediate' level of competitive asymmetries. Because the studied flock may represent a random sample of house sparrows with respect to their age, body condition etc., it probably included individuals of a wide range of competitive ability, so extreme differences in competitive ability might have been rare. According to the Barta \& Giraldeau (1998) model, we would expect more step-like changes in the tactic use if competitive asymmetries were increased experimentally, e.g. by removing some of the birds of intermediate ranks from the flock.

We did not detect a significant increase in feeding success among those individuals that joined more frequently or those of high dominance rank, although the direction of all non-significant results pointed toward such tendencies. It is unclear, however, what should specifically be expected for 
the studied flock by the phenotype limited model. Strong relationship s are predicted between dominance, tactic use and food intake rate only for high competitive asymmetries (Barta \& Giraldeau, 1998), which may not be the case for sparrows included in this study (see above). For intermediate levels of asymmetries the model predicts less marked differences (Fig. 7 in Barta \& Giraldeau, 1998), that may or may not include the weak relationships of tactic use and dominance with feeding rate we found in this study. On the other hand, the feeding advantages of dominants in ground feeding birds strongly depend on the distribution of food items, with the highest advantages occurring at the most patchily and most concentrated food distribution s (Rohwer \& Ewald, 1981; Theimer, 1987). We expect therefore that difference in feeding rate between dominant and subordinate house sparrows may also be larger when they forage on more concentrated food sources than those used in our study.

One may argue that Barta \& Giraldeau's (1998) model is not fully applicable to our system because the model assumes that individuals that joined obtain a share of the patch which is proportional to their dominance rank. In our system aggressive sparrows seem to displace finders so that the situation is closer to a 'get all or nothing' situation. Note, however, that the model does not specify how the sharing of food should happen. It can be a simultaneous process, i.e. all joined individuals get their share at the same time, but it can also occur as a sequential process, i.e. birds displace each other consecutively. Since the more dominant a bird, the more difficult can be to displace it, the actual partitioning of the patches in our system can be close to the one assumed by the model. The fact that we observed displacing individuals who was latter also displaced may support this argument.

The analyses of the behaviour of sparrows feeding on the grid showed that the locomotion frequency and vigilance behaviour of individuals were not related to their foraging tactic use or dominance. Finding no differences in the frequency of jumping probably reflect the fact that both dominants and subordinates should move a lot during feeding: subordinates have to find new food patches, whereas dominants have to follow them, in addition to finding new feeding sites on their own. Although some theoretical works (e.g. Ranta et al., 1996, 1998) predict that individuals using more scrounging should be more vigilant, a recent empirical study (Coolen et al., 2001) has shown that this may be true only for specific form of scanning behaviour, i.e. for head-up during movements. Coolen et al. (2001) suggest that other 
forms of vigilance (e.g. head-up while stationary) may have anti-predatory function. If individuals trade off these two forms of vigilant behaviour (headup-during-movement and head-up-while-stationary ) then the fact that we do not discriminate between these forms of vigilance during the analysis can explain the lack of relationship between vigilance and joining frequency found in this study. Finally, we found an increase in food searching behaviour in birds with low proportion of joining. This relationship s may reflect the fact that joining birds use other birds as cues to find food patches, and they have less time to investigate the potential unoccupied feeding places. Thus the two forms of food searching (finding and joining) may be incompatible and so our study system may fulfil one of the crucial assumptions of producerscrounger games (Giraldeau \& Caraco, 2000).

Beyond phenotype limitation of foraging tactics, our study has some general implications for social foraging. First, it clearly demonstrates that aggressive joining may represents the main method of scrounging in some social foraging systems. This contrasts most models (which assume individuals sharing a food patch without aggression), and highlight the need of new theoretical work to predict under which circumstances aggression will occur and how it will influence joining tendencies (Giraldeau \& Beauchamp, 1999). Second, the association between dominance and joining suggests that dominant house sparrows may use subordinates as food finders under some conditions. The shepherd hypothesis by Rohwer \& Ewald (1981) proposes that food finder subordinates are tolerated by dominants at good feeding places, and the benefits of the access to resources may explain why subordinates honestly signal their low status in birds where the costs of the signal are low (e.g. plumage badges). Although recent studies found that developing and maintaining a large black throat patch (the signal of dominant status) may involve some costs in house sparrows (Veiga \& Puerta, 1996; Poiani et al., 2000; Buchanan et al., 2001; but see Gonzalez et al., 2002), our results suggest that the mechanism proposed by the shepherd hypothesis may also be relevant to the maintenance of an honest badge signalling system in sparrows. The shepherd hypothesis has rarely been tested thoroughly in birds (Rohwer \& Ewald, 1981), and we suggest that house sparrows are particularly suitable to investigate the predictions of this interesting idea that link social foraging to communication in flock-feeding birds.

In conclusion, our study suggests that joining is related to dominance in house sparrows when they feed on rich and clumped food patches. The 
relationship between dominance and tactic use in sparrows is a gradual increase in joining frequency with increasing dominance, that resembles the pattern predicted for intermediate competitive asymmetries by a model of the problem (Barta \& Giraldeau, 1998). Predictions on food intake rate were not supported unambiguously, although our results may not necessarily disagree with the model. Further testing is necessary to determine whether finding events and joining events represent two incompatible searching strategies in house sparrows, such as producing and scrounging. The negative association found between joining and food searching rate of individuals nevertheless suggest that joining individuals may have reduced capabilities to detect unoccupied food patches, as it is assumed in a producer-scrounger system.

\section{References}

Baker, M.C., Belcher, C.S., Deutsch, L.C., Sherman, G.L. \& Thompson, D.B. (1981). Foraging success in junco flocks and the effects of social hierarchy. - Anim. Behav. 29 , p. 137-142.

Barnard, C.J. (1980). Flock feeding and time budget in the house sparrow (Passer domesticus L.). - Anim. Behav. 28, p. 295-309.

— — \& Sibly, R.M. (1981). Producers and scroungers: a general model and its application to captive flocks of house sparrows. - Anim. Behav. 29, p. 543-550.

Barta, Z. \& Giraldeau, L.-A. (1998). The effect of dominance hierarchy on the use of alternative foraging tactics: a phenotype-limited producing-scrounging game. - Behav. Ecol. Sociobiol. 42, p. 217-223.

Biondolillo, K., Stamp, C., Woods, J. \& Smith, R. (1997). Working and scrounging by zebra finches in an operant task. - Behav. Proc. 39, p. 263-269.

Buchanan, K.L., Evans, M.R., Goldsmith, A.R., Bryant, D.M. \& Rowe, L.V. (2001). Testosterone influences basal metabolic rate in male house sparrows: a new cost of dominance signalling? — Proc. R. Soc. Lond. B 268, p. 1337-1344.

Caraco, T., Barkan, C., Beacham, J.L., Brisbin, L., Lima, S., Mohan, A., Newman, J.A., Webb, W. \& Whithiam, M.L. (1989). Dominance and social foraging: a laboratory study. - Anim. Behav. 38, p. 41-58.

Coolen, I., Giraldeau, L.-A. \& Lavoie, M. (2001). Head position as an indicator of producer and scrounger tactics in a ground-feeding bird. - Anim. Behav. 61, p. 895-903.

Elgar, M.A. \& Catterall, C.P. (1981). Flocking and predator surveillance in house sparrows: a test of an hypothesis. - Anim. Behav. 29, p. 868-872.

Giraldeau, L.-A. \& Beauchamp, G. (1999). Food exploitation: searching for the optimal joining policy. - Trends Ecol. Evol. 14, p. 102-106.

— — \& Caraco, T. (2000). Social foraging theory. — Princeton, New Jersey.

— - , Hogan, J.A. \& Clinchy, M.J. (1990). The payoffs to producing and scrounging: What happens when patches are divisible? - Ethology 85, p. 132-146.

— — \& Livoreil, B. (1998). Game theory and social foraging. — In: Game theory and animal behavior (L.A. Dugatkin \& H.K. Reeve, eds). Oxford Univ. Press, New York, p. 16-37. 
— - Soos, C. \& Beauchamp, G. (1994). A test of the producer-scrounger foraging game in captive flocks of spice finches, Lonchura punctulata. - Behav. Ecol. Sociobiol. 34, p. 251-256.

Gonzalez, G., Sorci, G., Smith, L.C. \& de Lope, F. (2002). Social control and physiological cost of cheating in status signalling male house sparrows (Passer domesticus). Ethology 108, p. 289-302.

Johnson, C.A., Giraldeau, L.-A. \& Grant, J.W.A. (2001). The effect of handling time on interference among house sparrows foraging at different seed densities. - Behaviour 138 , p. 597-614.

Liker, A. \& Barta, Z. (2001). Male badge size predicts dominance against females in house sparrows. - Condor 103, p. 151-157.

Møller, A.P. (1987). Variation in badge size in male house sparrows Passer domesticus: evidence for status signaling. - Anim. Behav. 35, p. 1637-1644.

Motulsky, H. (1995). Intuitive biostatistics. — Oxford Univ. Press, New York.

Mottley, K. \& Giraldeau, L.-A. (2000). Experimental evidence that group foragers can converge on predicted producer-scrounger equilibria. - Anim. Behav. 60, p. 341-350.

Poiani, A., Goldsmith, A.R. \& Evans, M.R. (2000). Ectoparasites of house sparrows (Passer domesticus): an experimental test of the immunocompetence handicap hypothesis and a new model. - Behav. Ecol. Sociobiol. 47, p. 230-242.

Ranta, E., Peuhkuri, N., Laurila, A., Rita, H. \& Metcalfe, N.B. (1996). Producers, scroungers and foraging group structure. - Anim. Behav. 51, p. 171-175.

— - , — - Hirvonen, H. \& Barnard, C.J. (1998). Producers, scroungers and the price of a free meal. - Anim. Behav. 55, p. 737-744.

Rohwer, S. \& Ewald, P.W. (1981). The cost of dominance and advantage of subordination in a badge signalling system. - Evolution 35, p. 441-454.

Theimer, T.C. (1987). The effect of seed dispersion on the foraging success of dominant and subordinate dark-eyed juncos Junco hyemalis. — Anim. Behav. 35, p. 1883-1890.

Veiga, J.P. \& Puerta, M. (1996). Nutritional constraints determine the expression of a sexual trait in the house sparrow, Passer domesticus. - Proc. R. Soc. Lond. B 263, p. 229-234.

Wiley, H. (1991). Both high- and low-ranking white-throated sparrows find novel locations of food. - Auk 108, p. 8-15. 